\title{
Cost of three models of care for drug- resistant tuberculosis patients in Nigeria
}

Florence O. Bada ${ }^{1 *}$ D, Evaezi Okpokoro ${ }^{1}$, Nick Blok², Emmanuel Meribole ${ }^{3}$, Saswata Dutt ${ }^{1}$, Patrick Dakum,4, Alash'le Abimiku ${ }^{1,4}$, Alice Zwerling ${ }^{2,5}$ and Sandra V. Kik ${ }^{2,6}$

\begin{abstract}
Background: Nigeria accounts for a significant proportion of the global drug-resistant tuberculosis (DR-TB) burden, a large proportion of which goes untreated. Different models for managing DR-TB treatment with varying levels of hospitalization are in use across Nigeria, however costing evidence is required to guide the scale up of DR-TB care. We aimed to estimate and compare the costs of different DR-TB treatment and care models in Nigeria.

Methods: We estimated the costs associated with three models of DR-TB treatment and care: Model (A) patients are hospitalized throughout the 8-month intensive phase, Model (B) patients are partially hospitalized during the intensive phase and Model $(C)$ is entirely ambulatory. Costs of treatment, in-patient and outpatient care and diagnostic and monitoring tests were collected using a standardized data collection sheet from six sites through an ingredient's approach and cost models were based on the Nigerian National Tuberculosis, Leprosy and Buruli Ulcer Guideline - Sixth Edition (2014) and Guideline for programmatic and clinical management of drug-resistant tuberculosis in Nigeria (2015).

Results: Assuming adherence to the Nigerian DR-TB guidelines, the per patient cost of Model A was $\$ 18,528$ USD, Model B \$15,159 USD and Model C \$9425 USD. Major drivers of cost included hospitalization (Models A and B) and costs of out-patient consultations and supervision (Model C).

Conclusion: Utilizing a decentralized ambulatory model, is a more economically viable approach for the expansion of DR-TB care in Nigeria, given that patient beds for DR-TB treatment and care are limited and costs of hospitalized treatment are considerably more expensive than ambulatory models. Scale-up of less expensive ambulatory care models should be carefully considered in particular, when treatment efficacy is demonstrated to be similar across the different models to allow for patients not requiring hospitalization to be cared for in the least expensive way.
\end{abstract}

Keywords: Drug-resistant tuberculosis, Decentralized ambulatory care, Hospitalization, Cost

\section{Background}

Nigeria has the second highest burden of tuberculosis (TB) in Africa and is among the 30 high drug-resistant tuberculosis (DR-TB) burden countries with an estimated 4700 patients with drug-resistant-TB (DR-TB) in 2015. Of these, only 1241 DR-TB patients were identified and 656 placed on treatment in Nigeria [1]. The treatment gap for DR-TB patients remains large and may even be increasing as case detection improves with increased usage of the GeneXpert ${ }^{\circ}$ MTB/RIF technology

\footnotetext{
* Correspondence: fbada@ihvnigeria.org; badaflorence@gmail.com

${ }^{1}$ International Research Center of Excellence, Institute of Human Virology

Nigeria, 252 Herbert Macaulay Way, Central Business District, Abuja, Nigeria

Full list of author information is available at the end of the article
}

that allows for the rapid diagnosis of rifampicin resistant TB (RR-TB). This rapidly evolving landscape is a clarion call to policymakers and practitioners to respond with improvement and scale up of treatment delivery for DR-TB.

Nigeria commenced programmatic management of DR-TB (PMDT) in 2010 following approval by the Green Light Committee [2] and started with hospitalization of patients at specialized treatment centers for the entire 8-month intensive phase followed by decentralized ambulatory care for the 12-month continuation phase. In 2013, community PMDT was initiated with patients receiving their entire course of treatment via ambulatory care. This was in line with a wide-spread move towards reducing

(c) The Author(s). 2019 Open Access This article is distributed under the terms of the Creative Commons Attribution 4.0 International License (http://creativecommons.org/licenses/by/4.0/), which permits unrestricted use, distribution, and reproduction in any medium, provided you give appropriate credit to the original author(s) and the source, provide a link to the Creative Commons license, and indicate if changes were made. The Creative Commons Public Domain Dedication waiver (http://creativecommons.org/publicdomain/zero/1.0/) applies to the data made available in this article, unless otherwise stated. 
lengthy hospitalizations for DR-TB patients and promoting ambulatory care models where the patient can remain at home in the community during part of their treatment course. A systematic review comparing hospitalization with community based DR-TB care during the intensive phase suggested that both DR-TB models of care provide comparable treatment success rates, lending further support to the 2011 World Health Organization (WHO) recommendation as part of updated DR-TB guidelines to include ambulatory models for DR-TB treatment $[3,4]$.

Treating DR-TB is extremely costly [5], yet data remains limited around costs associated with various models of DR-TB care [6-9]. More accurate costs for treating DR-TB using various models are required for rational planning and allocation of resources, to determine optimal models of care and to prioritize competing health care issues.

Huge investments are needed to scale-up DR-TB care in Nigeria; either towards community systems' strengthening for utilization of ambulatory DR-TB treatment models or to scale-up infrastructure for hospital-based DR-TB models of care. The amount of resources required to achieve this are not readily available to the NTBLCP given an unfunded budget for TB control of 55\% in Nigeria in 2015 [1]. Thus, it is imperative that evidence be provided to guide investments towards the scale-up of the DR-TB program in Nigeria, while assuring the provision of high quality care for patients.

We performed a comprehensive cost analysis of the three different models of DR-TB treatment and care in Nigeria employed in the period from June 2013 through December 2014.

\section{Methods}

\section{Setting}

Nigeria has a per capita income of \$2178 USD [10] and an estimated DR-TB prevalence of 4.3\% among new and 25\% among retreatment TB cases [1]. Management of identified DR-TB cases is based on a standardized WHO approved treatment regimen of 20 months consisting of an 8-month intensive phase and a 12-month continuation phase. Patients are placed on pyrazinamide and four second-line anti-TB drugs namely levofloxacin, kanamycin (replaced by capreomycin when indicated), prothionamide and cycloserine. All five drugs are used for the 8-month intensive phase at the end of which kanamycin (or capreomycin) is discontinued for the remaining 12-month continuation phase.

Three models of DR-TB care were utilized in Nigeria between June 2013 and December 2014 and differed only in their 8-month intensive phase. Patients treated under Model A, were hospitalized for the complete duration of the intensive phase; patients in Model B were hospitalized for a duration of 5 months in the intensive phase while patients treated under Model $\mathrm{C}$ received the complete intensive phase treatment as ambulatory care in the community. The continuation phase was identical in each of the three models with directly observed treatment (DOT) provided daily in the community by a treatment supporter supplemented by visits to the DOT center every 2 weeks for drug pick-ups. Patients were also monitored via bi-weekly home visits by the specific DOT officer overseeing their care, monthly home visits by the TB and Leprosy Supervisor (TBLS) overseeing the local government in which they were receiving treatment, monthly home visits by the state DR-TB focal person and quarterly home visits by the state consilium which is a multi-disciplinary DR-TB team consisting of a state TB control officer, a state quality assurance officer who is usually also the laboratory focal person, the state logistics officer who also doubles as the state pharmacist, an ear, nose and throat (ENT) surgeon/ audiometrist, the state DR-TB focal person, a nurse from the treatment center or DOT center, a chest physician, a psychiatrist, a monitoring and evaluation focal person and a social worker.

Patients were assigned to one of the three models by health care professionals based on the patient's medical history, the availability of the model in their geographical region and the patient's health status at the time of commencing treatment taking into cognizance the patient's preference.

\section{Costing}

We performed a cost analysis to better understand the costs and important drivers of cost across the different models of DR-TB management. The cost analysis was performed from the perspective of the Nigerian NTP (health sector perspective) and includes all DR-TB related management costs such as diagnostic and monitoring tests, hospitalization, clinic visits, home visits, treatment supervision and drugs. We assumed strict adherence to the Nigerian NTBLCP Guideline for programmatic and clinical management of drug-resistant tuberculosis in Nigeria (2015) and no difference in the effectiveness of the treatment in the analysis (i.e. a cost minimization). The time horizon for the analysis was 20 months which is the length of a full course of DR-TB treatment. All costs were collected in NGN for the year 2014. Final costs are expressed in 2014 USD using the average exchange rate in 2014 of 1 USD $=158$ NGN [11]. Costs were not inflated to 2017 dollars due to the considerable change in exchange rate for the Naira between 2014 and 2017.

\section{Unit costs}

\section{Inpatient, outpatient and clinic costs}

Cost data were obtained from six health facilities; three treatment centers providing hospitalization as part of care and three DOT centers providing ambulatory DR-TB care. 
Costs were built using an ingredients approach, also called bottom-up approach whereby each resource or cost item required was identified and valued in order to calculate the unit cost. Costs were collected using a standardized data collection sheet at each site. To estimate the total cost of hospitalization, we averaged the costs from three treatment centers providing hospitalization as part of care. Likewise, to estimate the total cost of out-patient services, we averaged the costs from three DOT centers providing ambulatory DR-TB care.

Treatment center 1 is a secondary faith-based hospital in Nigeria's South-West Geopolitical zone, Treatment center 2 is a tertiary treatment and training center located in Nigeria's North West Geo-political zone and Treatment center 3 is a public secondary treatment center located in the South West Geo-political zone. Capital costs collected included equipment, vehicles and furniture and were annualized using a discounted rate of $3 \%$, and the assumption of 10 years of useful life. Information on staff salaries and grade levels were obtained from administrative documents and staff interviews. Cost for personnel and other recurrent costs, including utilities and maintenance costs, ancillary costs, including catering and laundry services, and the purchase, maintenance and operation of infrastructure and equipment were all collected from hospital expenditure and financial reports. Shared costs including salaries, furniture, supervision, transportation and vehicles were estimated through observation and interviews with health staff. In addition, costs for specific items were obtained from asset registers and procurement lists from different institutes supporting DR-TB care in Nigeria including Institute of Human Virology, Nigeria (IHVN), Damian Foundation and KNCV Tuberculosis Foundation. Total inpatient and outpatient costs were divided by the total number of inpatient days and outpatient visits to calculate the cost per inpatient day and outpatient visit, respectively.

Costs made in the community were collected from three DOT centers that provided ambulatory DR-TB care - DOT Center 1 was located in the South-South Geo-political zone, DOT Center 2 in the South-West Geopolitical zone and DOT Center 3 in the North-West Geo-political zone. Costs were obtained from hospital expenditure reports and interviews with staff. Outpatient services were assumed to use a proportion of hospital overhead costs [12] .

The time spent by each staff member of the state consilium on different types of visits to or from DR-TB patients or for related meetings, as well as the stipends given to these state staff for transportation and communication as they oversee the management of DR-TB patients, were obtained via staff interviews. Funds spent to convene meetings and for the transportation of staff for home visits were obtained from financial and meeting reports.

\section{Diagnostic and monitoring test costs}

Diagnostic and monitoring tests were performed as recommended in the NTBLCP management and control guidelines for the programmatic and clinical management of DR-TB. The costs of diagnostic and monitoring TB tests such as smear microscopy, Xpert, chest X-ray, culture (liquid and solid) and drug susceptibility testing (DST) were determined using an ingredients approach. This included costs of reagents and consumables, equipment, power supply and salaries of the laboratory personnel obtained from two laboratories; Laboratory 1 (Lab 1) is the National Reference Laboratory located in the North-West Geopolitical zone and Laboratory 2 (Lab 2) is a Private Laboratory stationed in the North Central Geo-political zone.

Salary costs were estimated in two ways; laboratory personnel were asked to estimate the time needed to perform a single test (or a batch of tests if appropriate) (bottom-up) and laboratory personnel performing each test were asked to estimate what proportion of their time they spent on each test (top-down) and both estimates were averaged. The cost of audiometric testing was obtained from the financial reports of three treatment centers. For chemistry and hematology tests, the actual costs were obtained from five commercial laboratories and averaged.

\section{Drug costs}

The drug costs were based on the Global Drug Facility price list which is the sole source for all the drugs used for DR-TB management in Nigeria.

\section{Frequencies}

Frequencies of visits, drugs used, tests, visits and hospital days in each of the 3 models were derived from the Nigerian national guideline for programmatic and clinical management of DR-TB in Nigeria (2015) (See appendix). For our analysis, we assumed strict adherence to the national guidelines even though we recognize that under routine circumstances this might not always be done.

Total health care costs for each model were calculated using the DR-TB cost-effectiveness analysis (CEA) -Tool (Version 1) which was developed by Management Sciences for Health (MSH) through the USAID-funded TB CARE 1 project and has been used and validated to determine health care costs for different DR-TB treatment options in Indonesia. [13]

The average costs for the diagnosis, treatment and care during the intensive and the continuation phases of treatment are described for each of the treatment models.

\section{Ethical issues}

The study spanned several states in the Federal Republic of Nigeria and entailed direct observation of health care workers (HCWs) as they cared for DR-TB patients as 
well as interviewing $\mathrm{HCW}$ s to obtain the required information to calculate the health service costs of providing care to DR-TB patients. Written informed consent was obtained from HCWs prior to interviewing them. The protocol was approved by the National Health Research Ethics Committee, Nigeria (NHREC).

\section{Results}

\section{Inpatient costs}

The costs constituting one bed day in a hospital for DR-TB patients are provided in Table 1. Costs for all components were consistently lower in Treatment Center 1 (\$22.91 USD) compared with Treatment Center 2 (\$87.67 USD) and 3 (\$49.0 USD), with Treatment Center 3 over two times as costly as Treatment Center 1 and Treatment Center 2 three times more costly for one hospital bed day than Treatment Center 1. Salaries were an important driver of cost across all hospitals (27.7, 25.8 and 42.2\%). Administrative costs were an important driver of cost for hospitals 2 and 3 (30.9 and 34.4\% respectively) while catering for patient's food (33\%) was the highest cost component for Treatment Center 1.

\section{Outpatient costs}

Costs for the different kinds of outpatient visits, conducted by the patient or the treatment supervisor(s) or teams varied slightly across the three DOT centers evaluated (Table 2). Salary costs were the main driver across all types of outpatient visits. Among the home visits, quarterly state team home visits were consistently the most costly outpatient visit ranging from \$132.89 USD to $\$ 248.90$ USD per visit with salary costs and costs for staff travel as main drivers of cost across all three States. Regular DOT provided at DOT centers during the intensive phase was consistently the least expensive outpatient visit ranging from $\$ 4.19$ to $\$ 4.76$ USD per visit across the three sites evaluated.

\section{Diagnostic and monitoring test costs}

Costs of TB tests were consistently higher in Lab 2 as compared to Lab 1 (Table 3). As expected [14], top down estimates for costing the contribution of staff salaries were usually higher compared with bottom up estimations. The cost for liquid culture (\$97.20 USD on average) was about three times as expensive as the cost for solid culture (\$39.43 USD on average). First line DST performed on liquid culture were the most expensive TB test cost ( $\$ 130.44$ USD on average). DST performed on solid culture, testing resistance against a panel of nine first and second line drugs were estimated to cost $\$ 68.23$ on average, but these costs could only be estimated in one lab since the other lab did not perform second line DST. An average of top down and bottom up costs were used in the analysis. For smear microscopy the cost of Ziehl-Neelsen and fluorescence were averaged.

Costs for other monitoring tests and the costs for individual TB medications are shown in Table 4.

The number of diagnostic and monitoring tests and drugs, as prescribed in the Nigerian NTBLCP DR-TB guideline, were identical for the three different models of care (Table 5). However, the models differed in the number of inpatient hospitalization days ( 243 for Model A, 152 for Model B and none for Model C) and type and frequency of the treatment monitoring visits during the intensive and continuation phases.

We estimated, that the total cost to provide the diagnostic and treatment care as outlined in the Nigerian DR-TB guidelines, would be $\$ 18,528$ USD per patient for Model A, \$15,159 USD per patient for Model B and $\$ 9425$ USD per patient for Model C (Fig. 1). For all models, the cost for patient care (either inpatient or outpatient) accounted for more than half of the total health service costs. Even though the frequency of home visits by the DOT officer or DR-TB focal person were substantially higher in the intensive phase for Model C, the total costs for home visits and out-patient visits (\$5806 USD)

Table 1 Costs for one bed day in a hospital for a DR-TB patient in USD

\begin{tabular}{|c|c|c|c|c|}
\hline Cost Component & Treatment Center 1 (\%) & Treatment Center 2 (\%) & Treatment Center 3 (\%) & Average (\%) \\
\hline Administration & $3.74(16.3)$ & $27.12(30.9)$ & $16.88(34.4)$ & $15.91(30)$ \\
\hline Transportation & $1.83(8.0)$ & $11.97(13.7)$ & $-{ }^{a}$ & $4.60(9)$ \\
\hline Laundry & $0.25(1.1)$ & $3.27(3.7)$ & $2.84(5.8)$ & $2.12(4)$ \\
\hline Radiology & $0.77(2.1)$ & $7.11(6.1)$ & $-\mathrm{b}$ & $2.62(5)$ \\
\hline Maintenance cleaning and security & $2.16(9.4)$ & $5.25(6.0)$ & $1.82(3.7)$ & $3.08(6)$ \\
\hline Catering & $7.57(33)$ & $9.46(10.8)$ & $6.31(12.9)$ & $7.78(15)$ \\
\hline Other supplies & $0.24(1.0)$ & $0.89(1.0)$ & $0.48(1.0)$ & $0.54(1)$ \\
\hline Salaries & $6.35(27.7)$ & $22.61(25.8)$ & $20.67(42.2)$ & $16.54(31)$ \\
\hline Total costs one bed day & $22.91(100)$ & $87.67(100)$ & $49.00(100)$ & $53.19(100)$ \\
\hline
\end{tabular}

${ }^{a}$ This treatment center had no official cars and no drivers so no costs could be attributed to transportation

${ }^{\mathrm{b}}$ This treatment center had no functioning radiological equipment during the period over which costing information was obtained; patients were referred out for chest X-rays when needed 
Table 2 Costs for outpatient consultations, home visits and treatment supervision in USD

\begin{tabular}{lllll}
\hline Cost Component & DOT 1 & DOT 2 & DOT 3 & Average (\%) \\
\hline Monthly clinic visits & & & & \\
Salary costs & 16.49 & 11.33 & 3.25 & $10.36(64)$ \\
Overhead & 3.1 & 1.95 & 0.63 & $1.89(12)$ \\
Transport & 3.16 & 3.16 & 3.16 & $3.16(19)$ \\
Other costs^ & 1.76 & 0.16 & 0.49 & $0.80(5)$ \\
Total & 24.51 & 16.60 & 7.53 & $16.21(100)$ \\
DOT at DOT Center - Intensive phase & & & \\
Salary costs & 0.88 & 0.58 & 1.17 & $0.88(20)$ \\
Overhead & 0.15 & 0.51 & 0.23 & $0.30(7)$ \\
Transport & 3.16 & 3.16 & 3.16 & $3.16(71)$ \\
Other costs^ & 0 & 0.15 & 0.2 & $0.12(3)$ \\
Total & 4.19 & 4.40 & 4.76 & $4.45(100)$
\end{tabular}

DOT at DOT Center - Continuation phase

\begin{tabular}{|c|c|c|c|c|}
\hline Salary costs & 0.88 & 0.58 & 1.17 & $0.88(20)$ \\
\hline Overhead & 0.15 & 0.51 & 0.23 & $0.33(7)$ \\
\hline Transport & 3.16 & 3.16 & 3.16 & $3.16(71)$ \\
\hline Other costs ${ }^{\mathrm{a}}$ & 0 & 0.15 & 0.2 & 0.12 (3) \\
\hline Total & 4.19 & 4.40 & 4.76 & $4.45(100)$ \\
\hline
\end{tabular}

Home visit by DOT Officer - Intensive Phase

\begin{tabular}{|c|c|c|c|c|}
\hline Salary costs (visit) & 4.03 & & 2.34 & $3.19(28)$ \\
\hline Salary costs (travel time) & 4.03 & & 3.50 & $3.77(34)$ \\
\hline Transport & 3.16 & & 3.16 & $3.16(28)$ \\
\hline Other costs ${ }^{a}$ & 1.60 & & 0.59 & $1.10(10)$ \\
\hline Total & 12.82 & $\mathrm{NA}+$ & 9.59 & $11.21(100)$ \\
\hline
\end{tabular}

Home visit by DOT Officer - Continuation Phase

\begin{tabular}{|c|c|c|c|c|}
\hline Salary costs (visit) & 4.03 & & 2.34 & $3.19(29)$ \\
\hline Salary costs (travel time) & 4.03 & & 3.50 & $3.77(34)$ \\
\hline Transport & 3.16 & & 3.16 & $3.16(28)$ \\
\hline Other costs ${ }^{a}$ & 1.60 & & 0.40 & $1.00(9)$ \\
\hline Total & 12.85 & $N A^{b}$ & 9.40 & $11.13(100)$ \\
\hline
\end{tabular}

Home visit by DRTB Focal Person

\begin{tabular}{|c|c|c|c|c|}
\hline Salary costs (visit) & 6.28 & & & $6.28(19)$ \\
\hline Salary costs (travel time) & 20.94 & & & $20.94(65)$ \\
\hline Transport & 3.16 & & & $3.16(10)$ \\
\hline Other costs ${ }^{\mathrm{a}}$ & 1.93 & & & $1.93(6)$ \\
\hline Total & 32.32 & $\mathrm{NA}+$ & $\mathrm{NA}+$ & $32.32(100)$ \\
\hline \multicolumn{5}{|l|}{ tome visit by TBL Supervisor } \\
\hline Salary costs (visit) & 2.01 & 2.01 & 2.09 & $2.04(20)$ \\
\hline Salary costs (travel time) & 4.03 & 4.03 & 6.28 & $4.78(46)$ \\
\hline Transport & 3.16 & 3.16 & 3.16 & $3.16(31)$ \\
\hline Other costs ${ }^{a}$ & 1.03 & 0.09 & 0 & $0.37(4)$ \\
\hline Total & 10.23 & 9.29 & 11.53 & $10.35(100)$ \\
\hline
\end{tabular}

Table 2 Costs for outpatient consultations, home visits and treatment supervision in USD (Continued)

\begin{tabular}{lllll}
\hline Cost Component & DOT 1 & DOT 2 & DOT 3 & Average (\%) \\
\hline Quarterly State Team Home visit & & & \\
Salary costs (visit) & 163.4 & 48.61 & 214.04 & $142.02(76)$ \\
Salary costs (travel time) & $0^{c}$ & 81.03 & 26.76 & $35.93(19)$ \\
Transport & 3.16 & 3.16 & 3.16 & $3.16(2)$ \\
Other costs & \\
Total & 10.04 & 0.09 & 4.94 & $5.02(3)$ \\
Consilium Meeting $_{\text {Salary }}$ & 176.60 & 132.89 & 248.90 & $186.13(100)$ \\
Allowances & & & & \\
Catering & 58.48 & 26.81 & 49.51 & $44.93(75)$ \\
Other costs $^{\mathrm{a}}$ & 0 & 9.04 & 20.1 & $9.71(16)$ \\
Total & 0 & 5.42 & 0 & $1.81(3)$ \\
\hline
\end{tabular}

DRTB drug resistant tuberculosis, NA not available, TBL tuberculosis

and leprosy

${ }^{a}$ Other costs include the cost of supplies such as gloves, face masks, N-95 respirators, hand sanitizer, syringes and needles and spirit swabs

${ }^{\mathrm{b}}$ No available data for DOT Officer and DR-TB Focal Person during the data collection exercise in their states

It was not possible to separate travel time from visit time; therefore both are captured under Salary costs (visit)

were still much lower than those for the total or partial inpatient hospitalization in the intensive phase in Models A (\$12,925 USD) and B (\$8085 USD).

\section{Discussion}

We performed this cost analysis to calculate the total costs associated with the management of DR-TB in Nigeria from the health sector perspective and the costs of individual components of DR-TB care including the costs for hospitalization and outpatient costs, costs of visits to the DOT center, home visits, treatment supervision, drug costs and costs of diagnostic and monitoring tests. To our knowledge, this is the first study that has quantified the costs of treating DR-TB in Nigeria utilizing different models of care according to the 2015 Nigerian DR-TB guidelines. Results from this study will facilitate rational planning and resource allocation as Nigeria scales up DR-TB care including ambulatory models of treatment delivery and the introduction of shorter DR-TB Treatment regimen, new TB drugs such as Delamanid and Bedaquiline as well as repurposed drugs like linezolid and clofazimine.

Our main findings showed that Model A with hospitalization for the entire 8-month intensive phase cost 18528 USD (equivalent to $2,927,464$ NGN in 2014) per treated patient, two times the cost as estimated for Model C (9425USD, equivalent to $1,489,080$ NGN) where patients are managed through decentralized ambulatory care. The cost for Model B with hospitalization for 5 months during the intensive phase was 15159USD 
Table 3 Costs of tuberculosis tests in USD

\begin{tabular}{|c|c|c|c|c|c|}
\hline & \multicolumn{2}{|l|}{ Lab 1} & \multicolumn{2}{|l|}{ Lab 2} & \multirow[t]{2}{*}{ Average (\%) } \\
\hline & Salaries bottom-up & Salaries top-down & Salaries bottom-up & Salaries top-down & \\
\hline \multicolumn{6}{|c|}{ Cost of 1 AFB smear microscopy Ziehl-Neelsen } \\
\hline Reagents & 0.33 & 0.33 & 0.43 & 0.43 & $0.38(6)$ \\
\hline Supplies & 1.36 & 1.36 & 4.90 & 4.90 & $3.13(49)$ \\
\hline Labor & 0.91 & 1.74 & 1.55 & 1.23 & $1.36(21)$ \\
\hline Equipment & 0.70 & 0.70 & 0.99 & 0.99 & $0.85(13)$ \\
\hline Overhead & 0.36 & 0.36 & 0.87 & 0.87 & $0.62(10)$ \\
\hline Total & 3.67 & 4.50 & 8.73 & 8.41 & $6.33(100)$ \\
\hline \multicolumn{6}{|c|}{ Cost of 1 AFB Smear microscopy fluorescence } \\
\hline Reagents & 0.30 & 0.30 & 0.30 & 0.30 & $0.30(5)$ \\
\hline Supplies & 1.41 & 1.41 & 4.90 & 4.90 & $3.16(20)$ \\
\hline Labor & 1.00 & 1.74 & 1.66 & 1.23 & $1.41(22)$ \\
\hline Equipment & 0.72 & 0.72 & 0.99 & 0.99 & $0.86(13)$ \\
\hline Overhead & 0.38 & 0.38 & 0.86 & 0.86 & $0.62(10)$ \\
\hline Total & 3.80 & 4.55 & 8.72 & 8.28 & $6.34(100)$ \\
\hline \multicolumn{6}{|c|}{ Cost of solid culture ${ }^{a}$} \\
\hline Reagents & 12.04 & 12.04 & 13.73 & 13.73 & 12.89 (33) \\
\hline Supplies & 14.40 & 14.40 & 5.07 & 5.07 & $9.74(25)$ \\
\hline Labor & 0.95 & 5.31 & 4.84 & 14.67 & $6.44(16)$ \\
\hline Equipment & 6.40 & 6.40 & 8.57 & 8.57 & $7.49(19)$ \\
\hline Overhead & 3.72 & 3.72 & 2.03 & 2.03 & $2.88(7)$ \\
\hline Total & 37.51 & 41.87 & 34.24 & 44.08 & $39.43(100)$ \\
\hline \multicolumn{6}{|c|}{ Cost of liquid culture } \\
\hline Reagents & 12.00 & 12.00 & 14.84 & 14.84 & $13.42(14)$ \\
\hline Supplies & 27.65 & 27.65 & 20.21 & 20.21 & $23.93(25)$ \\
\hline Labor & 1.02 & 6.48 & 3.64 & 48.48 & $14.91(15)$ \\
\hline Equipment & 22.35 & 22.35 & 52.25 & 52.25 & $37.30(38)$ \\
\hline Overhead & 6.93 & 6.93 & 8.37 & 8.37 & $7.65(8)$ \\
\hline Total & 69.94 & 75.41 & 99.31 & 144.15 & $97.20(100)$ \\
\hline \multicolumn{6}{|c|}{ Cost of first line DST (liquid culture) } \\
\hline Reagents & 9.59 & 9.59 & 9.50 & 9.50 & $9.55(7)$ \\
\hline Supplies & 43.42 & 43.42 & 61.57 & 61.57 & $52.50(40)$ \\
\hline Labor & 0.88 & 20.28 & 3.02 & 73.90 & $24.52(19)$ \\
\hline Equipment & 16.43 & 16.43 & 50.88 & 50.88 & $33.66(26)$ \\
\hline Overhead & 7.73 & 7.73 & 12.70 & 12.70 & $10.22(8)$ \\
\hline Total & 78.05 & 97.45 & 137.68 & 208.56 & $130.44(100)$ \\
\hline \multicolumn{6}{|c|}{ Cost for first and second line DST (solid culture) } \\
\hline Reagents & 3.44 & 3.44 & & & $3.44(5)$ \\
\hline Supplies & 41.52 & 41.52 & & & $41.52(61)$ \\
\hline Labor & 1.42 & 28.39 & & & $14.90(22)$ \\
\hline Equipment & 2.94 & 2.94 & & & $2.94(4)$ \\
\hline Overhead & 5.43 & 5.43 & & & $5.43(8)$ \\
\hline Total & 54.74 & 81.71 & ND & ND & $68.23(100)$ \\
\hline
\end{tabular}


Table 3 Costs of tuberculosis tests in USD (Continued)

\begin{tabular}{|c|c|c|c|c|c|}
\hline & \multicolumn{2}{|l|}{ Lab 1} & \multicolumn{2}{|l|}{ Lab 2} & \multirow[t]{2}{*}{ Average (\%, } \\
\hline & Salaries bottom-up & Salaries top-down & Salaries bottom-up & Salaries top-down & \\
\hline \multicolumn{6}{|c|}{ Cost of Xpert MTB/RIF test } \\
\hline Reagents & 9.98 & 9.98 & 9.98 & 9.98 & $9.98(41)$ \\
\hline Supplies & 1.28 & 1.28 & 8.93 & 8.93 & $5.11(21)$ \\
\hline Labor & 0.57 & 11.30 & 0.56 & 11.23 & $5.92(24)$ \\
\hline Equipment & 2.28 & 2.28 & 3.49 & 3.49 & $2.89(12)$ \\
\hline Overhead & 1.55 & 0.01 & 1.43 & 0.01 & $0.75(3)$ \\
\hline Total & 15.67 & 24.85 & 24.38 & 33.64 & $24.64(100)$ \\
\hline
\end{tabular}

List of abbreviations: AFB Acid-fast bacilli, DST drug susceptibility testing, MTB Mycobacterium tuberculosis, ND not done in this lab, RIF rifampicin, TB tuberculosis

${ }^{a}$ solid culture based on Lowenstein Jensen media

(equivalent to 2,395,070 NGN). Managing patients without hospitalization reduces the cost of DR-TB care by approximately $49 \%$ in the Nigerian setting. This is in line with findings from similar studies $[15,16]$, which showed that models with hospitalization were more costly compared to partial or fully ambulatory treatment models.

Table 4 Costs of ancillary monitoring tests and DR-TB medications in USD

\begin{tabular}{|c|c|}
\hline & Cost per Unit \\
\hline \multicolumn{2}{|l|}{ Ancillary monitoring tests } \\
\hline Audiometry & 31.65 \\
\hline Kidney function tests $(E, U, C r)$ & 21.04 \\
\hline Thyroid Function Test & 62.18 \\
\hline Urinalysis & 2.69 \\
\hline Liver Function Test & 22.26 \\
\hline Fasting Blood Sugar & 4.85 \\
\hline Chest X-Ray & 10.92 \\
\hline HIV Test & 6.33 \\
\hline Pregnancy test & 2.85 \\
\hline \multicolumn{2}{|l|}{ DR-TB medications } \\
\hline Capreomycin $1 \mathrm{~g}$ (per vial) & 4.7 \\
\hline Amikacin 500 mg (per vial) & 0.62 \\
\hline Cycloserine 250 mg (per capsule) & 0.29 \\
\hline Ethambutol 400 mg (per tab) & 0.03 \\
\hline Prothionamide 250 mg (per tab) & 0.07 \\
\hline Kanamycin $1 \mathrm{~g}$ vial (per vial) & 0.24 \\
\hline Levofloxacin 250 mg (per caplet) & 0.03 \\
\hline Pyrazinamide 400 mg (per tab) & 0.02 \\
\hline Pyrazinamide 500 mg (per tab) & 0.03 \\
\hline Pyridoxin 10 mg (per tab) & 0.03 \\
\hline
\end{tabular}

Cr creatinine, DR-TB drug resistant tuberculosis, DST drug susceptibility testing, HIV human immunodeficiency virus, $E$ electrolytes, $U$ urea

The cost of audiometric testing was obtained from the financial reports of three treatment centers. For C-Xrays, chemistry and hematology tests, the actual costs were obtained from five commercial laboratories and averaged. The drug costs were based on the Global Drug Facility price list
Hospitalization was responsible for over $70 \%$ of the cost of Model A while out-patient consultations and supervision and laboratory diagnostic and follow-up tes ting contributed 9 and $12 \%$ respectively. However, the level of treatment facility utilized for treatment (secondary or tertiary) significantly affected the cost of hospita lization with hospitalization in a tertiary hospital being more than twice the cost of hospitalization in a secondary public hospital and three times the cost of hospitalization in a secondary faith-based hospital according to our findings. This may be explained by the fact that treatment center 1 was a faith-based hospital with minimal support from the government and thus has less funds available for salaries and overhead costs while treatment center 2 which is supported by the Federal Government of Nigeria and treatment center 3 by a State Government are well funded with salaries and administrative costs associated with these two hospitals notably higher. Transportation costs in treatment center 2 accounted for $13.7 \%$ of the total hospitalization costs due to many vehicles owned and used by the tertiary hospital as well as the costs of fueling and maintaining the vehicles.

For Model C, outpatient consultations and supervision were responsible for over $62 \%$ of the cost, while laboratory diagnostic and monitoring tests accounted for $28 \%$. These cost drivers are similar to those found in other studies $[6,16]$ with the exception of drugs which only contributed $5-9 \%$ across the three models in this study. Costs for the monthly clinic visits varied the most across the different states, mainly due to the different number and cadre of staff participating in these visits and the time spent with the patient during these visits. Quarterly state team home visits were consistently the most costly outpatient visit, ranging from \$133USD to \$249USD per visit with salary costs and costs for staff travel as main drivers across all three states and greatly influenced by the time spent journeying to the patients' residences. Regular DOT provided at DOT centers during the intensive phase was consistently the least expensive outpatient visit ranging from $\$ 4.19$ to $\$ 4.76$ USD per visit across the three 
Table 5 Frequencies of laboratory tests and patient monitoring visits per patient and model according to the Nigerian DR-TB guidelines

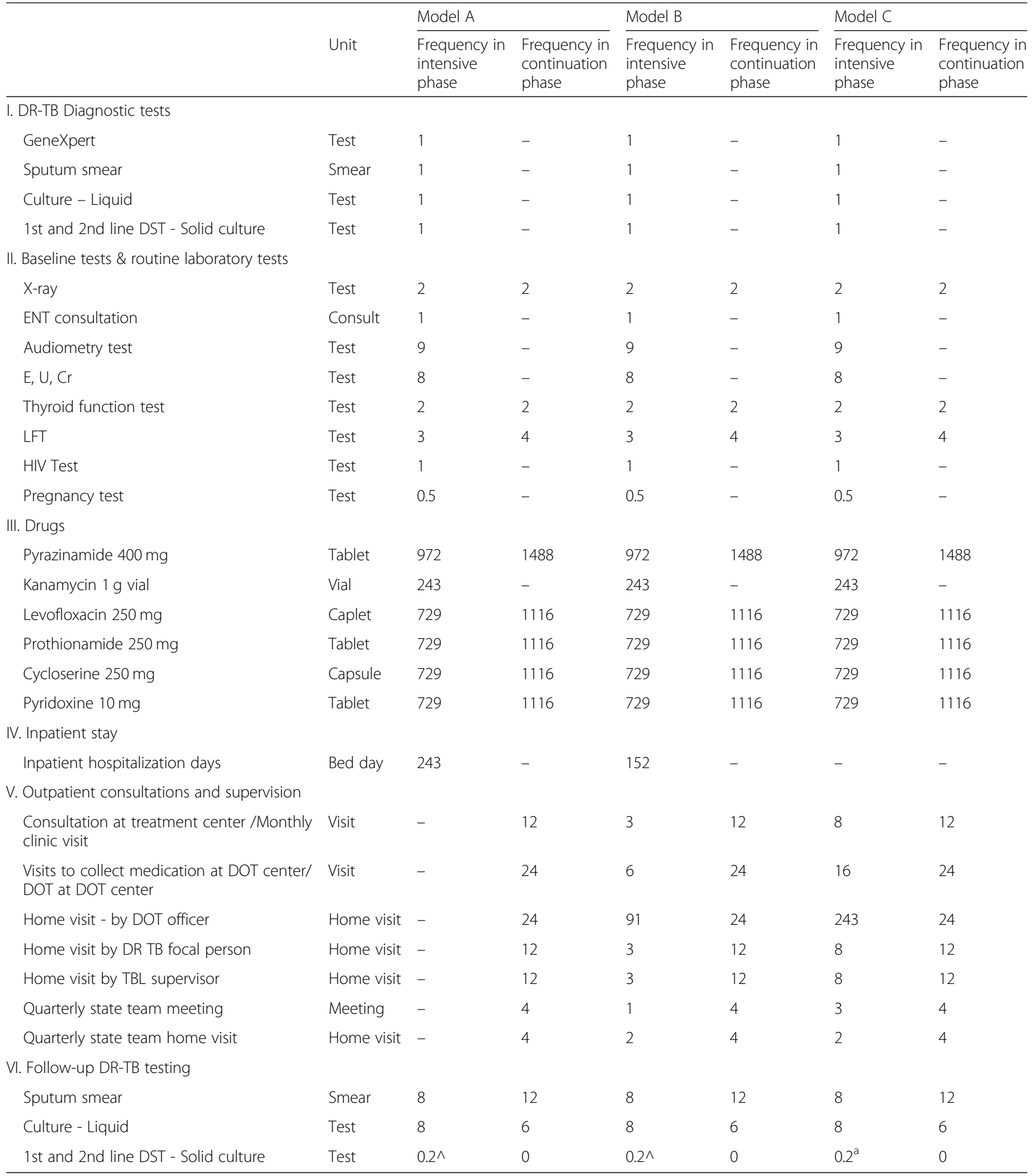

List of abbreviations: $\mathrm{Cr}$ creatinine, DOT directly observed treatment, $D R$ drug resistant, DST drug susceptibility testing, $E$ electrolytes, ENT ear nose throat, $T B$ tuberculosis, TBL tuberculosis and leprosy, $U$ urea, $L F T$ liver function test,

${ }^{a} \mathrm{~A}$ conservative estimate of DR-TB patients who are still culture positive after four months of DR-TB treatment and thus require repeat second-line DST

sites evaluated and should continue to be promoted as a feasible way to ensure optimal patient monitoring. However, we did not take the patient costs associated with these outpatient visits into account. Home visits by $\mathrm{HCW}$ though slightly more expensive can continue to be interspersed with patient visits to the DOT center and have the 


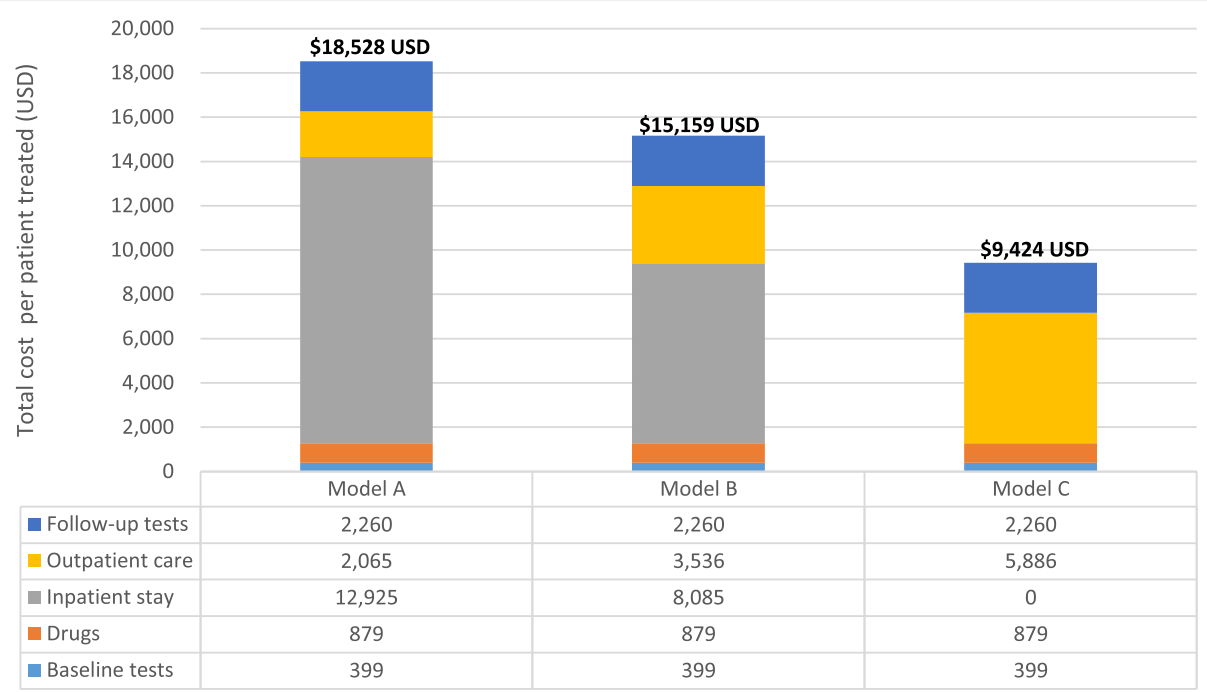

Fig. 1 Total Costs for three models of DR-TB treatment care in Nigeria

added advantage of providing a means for health care workers to ensure conditions in the patient's home minimize the risk of TB transmission to family members and neighbors. The significant contribution of out-patient consultation and supervision to ambulatory models, emphasize findings from other studies that when shifting from hospital-based care to ambulatory systems, provision needs to be made for alternate health system support [17].

While outpatient consultations were more expensive for Models B and C with ambulatory care, these increased costs were still dwarfed by costs associated with hospitalization in Model A. The use of decentralized models have the added benefit of earlier treatment initiation and preserving the patient's social support structure and are thought to have comparable success rates to models with some degree of hospitalization [3].

Salary costs are significant drivers of cost in all components of DR-TB care therefore, particularly among outpatient consultation and monitoring, optimizing human resources in these activities is critical in future scale-up activities. Exploring task shifting of patient supervision may increase coverage of patient supervision and yield considerable cost savings.

There are a number of limitations to our study. We were not able to include building costs which are a substantial cost because land for the construction of government hospitals is not purchased but appropriated for the purpose and there are no standard rates for renting per square meter which could have served as a proxy for building costs. We were also not able to include costs for ancillary medications given to patients and general supplies utilized by patients while hospitalized because although the total costs spent on ancillary medication and hospital supplies were obtained for each treatment center, insufficient utilization information was available to allocate proportionate costs to DR-TB patients hospitalized at the treatment centers in these health facilities.

Frequency of diagnostic and monitoring tests and outpatient consultations were all based on the 2015 Nigerian DR-TB guidelines, while in reality individual patients may undergo more or fewer tests and visits depending on their situation. We solely described the cost for the complete treatment of a patient with DR-TB when cared for under one of three models of DR-TB care available in Nigeria, without comparing the effectiveness of the different models. During the study period, DR-TB patients were allocated to a model based on availability and the preference of the patient and clinician. Severely ill patients might have been assigned more often to model A based on their clinical condition. Additional studies are needed to build evidence around the most cost-effective model for different patients taking their disease characteristics and clinical condition into account. The cost-effectiveness of each of the models will be published in a later study which will take into account actual frequencies of visits and resources used by individual patients.

The use of cost data from treatment centers housed in three different types of hospitals might not reflect the whole of Nigeria. However, hospitals were selected from different geographical zones to achieve a representative picture of DR-TB costs and ranged from a National Tertiary hospital to a small district hospital.

The strengths of the study include the evaluation of three treatment models within the same geopolitical setting and the collection of detailed data used to build unit costs for Nigeria instead of using previously published estimates or projections from other settings. 


\section{Conclusion}

This costing study demonstrates that DR-TB treatment models employing ambulatory care are considerably less costly compared to approaches that utilize hospitalization. Scale-up of less expensive ambulatory care models should be carefully considered in particular, when treatment efficacy is demonstrated to be similar across the different models to allow for patients not requiring hospitalization to be cared for in the least expensive way in Nigeria to address the large treatment gap in treating DR-TB patients.

\section{Abbreviations}

AFB: Acid-fast bacilli; CEA: Cost-effectiveness analysis; $\mathrm{Cr}$ : Creatinine; DOT: Directly observed treatment; DR-TB: Drug-resistant tuberculosis; DST: Drug susceptibility testing; E: Electrolytes; ENT: Ear, nose and throat; HCW: Health care worker; HIV: Human immunodeficiency virus; LFT: Liver function test; MSH: Management sciences for health; MTB: Mycobacterium tuberculosis; NA: not available; ND: not done; NGN: Nigerian naira; NTBLCP: National tuberculosis and leprosy control programme; NTP: National tuberculosis programs; PMDT: Programmatic management of drug-resistant TB; RIF: Rifampicin; TB: Tuberculosis; TBL: Tuberculosis and leprosy; TBLS: Tuberculosis and leprosy supervisor; U: Urea; USD: United States dollars; WHO: World Health Organization

\section{Acknowledgements}

We thank the past and present National Coordinators of the Nigerian NTBLCP - Dr. Gabriel Akang and Dr. Adebola Lawanson for spearheading the implementation of this research and facilitating buy-in from state, local government and facility-based staff of the TB programme. We thank the state, local government and facility-based staff for their invaluable collaboration. We thank Dr. Mustapha Gidado for his kind assistance and guidance on this project. We thank Drs. Aderonke Agbaje, Vivian Ibeziako and Nicaise Ndembi for their guidance as this project was conceptualized and implemented. We appreciate Drs. M. Yashim-Ankut, Olayemi Olupitan and Linda Arogundade for data-collection in the field. We thank Joseph Kuye and Emmanuel Opeyemi who helped retrieve data from the National Electronic TB database. We thank staff at the International Research Center of Excellence, IHVN for their support to this research.

\section{Funding}

This work was supported by the Global Fund New Funding Model MDR-TB grant to Nigeria with IHVN serving as the principal recipient. The funder had no role in the design, data collection, analysis and interpretation of results in this study.

\section{Availability of data and materials}

The datasets used and/or analyzed during the current study are available from the corresponding author on reasonable request.

\section{Authors' contributions}

$F B, E O, N B, S K, S D, P D$ conceived the study, FB, EO, NB, SK, EM, AZ contributed to data collection, FB, EO, AZ analyzed the data, NB, SK,SD, EM, AA critically reviewed the results, $F B, E O, N B, A Z$, SK, drafted the manuscript. All authors read and approved the final manuscript.

\section{Ethics approval and consent to participate}

Ethical approval was obtained from the Nigerian National Health Research Ethics Committee, under number NHREC/01/01/2007-29/09/2015b. No consent from individual patients was required for this study. The study entailed direct observation of HCWs as they cared for DR-TB patients as well as interviewing HCWs to obtain the required information to calculate the health service costs of providing care to DR-TB patients. Written informed consent was obtained from HCWs prior to interviewing them.

\section{Competing interests}

The authors declare that they have no competing interests.

\section{Publisher's Note}

Springer Nature remains neutral with regard to jurisdictional claims in published maps and institutional affiliations.

\section{Author details}

${ }^{1}$ International Research Center of Excellence, Institute of Human Virology Nigeria, 252 Herbert Macaulay Way, Central Business District, Abuja, Nigeria. ${ }^{2}$ KNCV Tuberculosis Foundation, The Hague, The Netherlands. ${ }^{3}$ Department of Health, Planning, Research and Statistics, Federal Ministry of Health, Abuja, Nigeria. ${ }^{4}$ University of Maryland School of Medicine, Baltimore, MD, USA. ${ }^{5}$ School of Epidemiology and Public Health, University of Ottawa, Ottawa, Canada. ${ }^{6}$ Academic Medical Center, Amsterdam Institute for Global Health and Development, Amsterdam, Netherlands.

Received: 27 November 2017 Accepted: 19 December 2018

Published online: 10 January 2019

\section{References}

1. WHO. "Global tuberculosis report 2016," Cdc 2016, no. Global TB Rep. 2016; 2016:214.

2. Gupta R, et al. Increasing transparency in partnerships for health introducing the green light committee. Trop Med Int Health. 2002;7(11): 970-6.

3. Bassili A, Fitzpatrick C, Qadeer E, Fatima R, Floyd K, Jaramillo E. Review article: a systematic review of the effectiveness of hospital and ambulatorybased management of multidrug-resistant tuberculosis. Am J Trop Med Hyg. 2013;89(2):271-80.

4. World Health Organization. Guidelines for the programmatic management of multidrug-resistant. Tuberculosis. 2011. Available from: http://apps.who. int/iris/bitstream/handle/10665/44597/9789241501583_eng.pdf;jsessionid= DE0961DED99BD90C047D67790398250A? sequence $=1$.

5. Pooran A, Pieterson E, Davids M, Theron G, Dheda K. What is the cost of diagnosis and Management of Drug Resistant Tuberculosis in South Africa? PLoS One. 2013:8(1)

6. Fitzpatrick C, Floyd K. A systematic review of the cost and cost effectiveness of treatment for multidrug-resistant tuberculosis. Pharmacoeconomics. 2012; 30(1):63-80

7. Resch SC, Salomon JA, Murray M, Weinstein MC. Cost-effectiveness of treating multidrug-resistant tuberculosis. PLoS Med. 2006;3(7):1048-57.

8. Tupasi TE, et al. Feasibility and cost-effectiveness of treating multidrugresistant tuberculosis: a cohort study in the Philippines. PLoS Med. 2006;3(9): 1587-96.

9. Laurence $\mathrm{W}$, Griffiths UK, Vassall A. Costs to health services and the patient of treating tuberculosis: a systematic literature review. Pharmacoeconomics. 2015:33(9):939-55

10. W. Bank, "GDP Per Capita Data," GDP Per Capita, 2016. [Online]. Available: https://data.worldbank.org/indicator/NY.GDP.PCAP.CD?locations=NG. [Accessed: 05-Nov-2017]

11. W. Bank, "Nigeria Official Exchange Rate," 2014. [Online]. Available: https:// data.worldbank.org/indicator/PA.NUS.FCRF?end=2016\&locations=NG\&name_ desc $=$ true\&start=1960\&view=chart

12. World Health Organization, "Guidelines for costs and cost-effectiveness analysis of tuberculosis control," 2002.

13. MSH, "TB Care 1 Costing Tools." [Online]. Available: http://www.msh.org/ resources/multidrug-resistant-tuberculosis-mdr-tb-cost-effectiveness-analysistool.

14. Rupert S, et al. Bottom-up or top-down: unit cost estimation of tuberculosis diagnostic tests in India. Int J Tuberc Lung Dis. 2017;21(4):375-80.

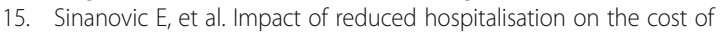
treatment for drug-resistant tuberculosis in South Africa. Int J Tuberc Lung Dis. 2015;19(2):172-8.

16. Marks SM, Salcedo K, Graviss EA, Oh P, Seaworth B, Flood J. "Characteristics and costs of multidrug-resistant tuberculosis in- patient care in the United States, 2005-2007," vol. 20, no. October 2015; 2016. p. 435-41.

17. Kohler $\mathrm{S}$, et al. Health system support and health system strengthening: two key facilitators to the implementation of ambulatory tuberculosis treatment in Uzbekistan. Health Econ Rev. 2016:6(1):28. 